\title{
Incentivizing Selected Devices to Perform Cooperative Content Delivery: A Carrier
} Aggregation based Approach

Bidushi Barua, Zaheer Khan, Zhu Han, Alhussein A. Abouzeid, and Matti Latva-aho

\begin{abstract}
In cooperative content distribution (CCD) using multiple interfaces, a smart wireless device receives contents from a base station on its cellular interface, and it broadcasts the same contents through another wireless interface, such as WiFi. However, different users can experience different link qualities, and users with slow wireless links can be a bottleneck in terms of CCD performances. To address this problem, we propose a device selection method which leverages multiple interfaces of the selected devices to perform CCD. Our proposed method takes into account the link quality of both the primary (cellular) and secondary (WiFi/short-range) interfaces of the devices, and selects the devices with the best link quality for CCD. To analyze the stability of the proposed method against selfish deviators, we model the problem as a repeated CCD game. We show that although the proposed method yields significant gains in terms of frequency carrier and energy saving performances, it is vulnerable to selfish deviating users. To address this challenge, we propose a carrier aggregation based incentive mechanism for the proposed method. The analytical and simulation results show that the proposed mechanism maximizes individual and network payoffs, and is an equilibrium against unilateral selfish deviations.
\end{abstract}

\section{Index Terms}

Cooperative content delivery, multiple wireless interfaces, off-loading, incentive mechanism, carrier aggregation and game theory.

\section{INTRODUCTION}

Over the last few years the surge in new smart device users has led to a phenomenal growth in mobile/cellular data traffic. As the developing world is expected to add more than $400 \mathrm{~m}$ new users of smart devices to the global network, the growth in demand for data traffic is expected to continue [1], [2].

B. Barua, Z. Khan, and Matti Latva-aho are with the Centre for Wireless Communications, University of Oulu, Finland.

Z. Han is with the University of Houston, TX, USA, and A. A. Abouzeid is with the Rensselaer Polytechnic Institute, Troy, NY, USA

This work was funded by the Academy of Finland under the WIFIUS program.

Preliminary results from this paper were presented in part at the IEEE Global Communications Conference (GLOBECOM'14), 2014. [Online]. Available: http://www.ee.oulu.fi/ zaheer/Globecom2014.pdf. 
To address this challenge the wireless industry is preparing for a long term 1000 times more data traffic in cellular networks [3], [4]. Though smart devices are content hungry, over the years, these devices have exhibited advanced features, such as support for large memory space, increasing processing capabilities, and also support for using multiple interfaces (such as cellular and WiFi).

In the current cellular networks, the default method of distributing content among users is by independent downloading of the requested content by each user using their own cellular links [5]. This currently used default method of operation can lead to cellular traffic congestion in scenarios where many users (that are in proximity of one another) demand rich content applications. The availability of multiple wireless interfaces on smart devices could potentially enable these devices to operate in heterogeneous environments and use diverse protocols, such as cellular and WLAN protocols. These features have attracted the interest of researchers to use multiple wireless interfaces in the context of cooperative content delivery (CCD). For instance, the works in [1], [2], [6], [7] have shown that the problem of congestion can be mitigated if the available multiple interfaces on smart devices are utilized intelligently to disseminate data contents among a group of users that are in the vicinity of one another.

Several practical use cases of CCD have been discussed in different works, a few of which include (but are not limited to): 1) Devices that simultaneously request the same data content. For instance, when a group of devices want to watch a video (such as a live game or a popular YouTube content) simultaneously. It is in general not comfortable for more than one user to watch the video together on one phone/tablet screen; 2) Devices that asynchronously request for the same popular content. For instance, a group of people who are interested in watching on their devices the same popular audio/video content and the news clip but usually at different times; and 3) Software updates on smart mobile devices at regular intervals of time. The practical use cases of CCD using multiple interfaces for a group of smart phones at close proximity, can also be found in areas beyond the field of entertainment. Documentary videos and educational movies can enhance the learning process adopted by educators. Moving images are in general more attractive for students as compared to texts alone. This had led to the fast paced advancement in the field of mobile learning [8].

The intelligent design of CCD methods exploiting multiple interfaces on devices, is a powerful solution to overcome the limitations of traditional content delivery methods using single interface of mobile devices. Several works have shown that, apart from dealing with the problem of cellular traffic congestion, CCD methods using multiple interfaces have led to carrier frequency resource savings and also more energy efficiency in wireless networks [9]-[13]. In this paper, we study CCD using multiple interfaces for 
scenarios in which two or more devices are in the vicinity of one another and request the same content simultaneously. We consider a model where a device receives the requested content from a base station on its cellular interface and broadcasts the same content to other devices in its vicinity through another wireless interface, such as WiFi. Design of the CCD techniques that use multiple network interfaces is an actively researched topic [11]- [12]. However, in much of the existing literature, the secondary wireless interface links of all the cooperating devices are considered to be similar in terms of transmit/receive performances. In reality link qualities are different. Moreover, due to the broadcast shared nature of the secondary link medium, a single user with slow bitrate or due to poor channel quality or low signal from other users can serve as a bottleneck in terms of CCD performance (such as in WiFi). To address this challenge, we present a method for intelligent selection of devices for wireless content distributions. The proposed method takes into account the link qualities of both the primary (cellular) and the secondary link (WiFi/ short-range) network interfaces, and performs selection of those devices for CCD that have good primary links with the base station, and also good secondary links to other devices.

The main contributions of this paper are:

- We propose a method called Select Best (SB) to perform selection of devices for CCD. Our proposed device selection method takes into account the quality of both primary (cellular) and secondary (WiFi) links of users that are interested in the same content. The proposed method incurs little overhead as it utilizes information (such as acknowledgments of content packets) for device selection, which already exists in the network.

- We evaluate the performance of the proposed method in terms of: 1) Number of carriers utilized by the cellular BS; 2) Average bits-per-Joule performance; 3) The average time required to deliver a content file to all the users. We compare the proposed method against the methods in which: 1) Only primary (cellular) link interface is utilized to deliver content to all the users; 2) The BS selects the devices for CCD, but in this case selection is performed while taking into account only the cellular link quality of the devices. Moreover, in our performance evaluation, we also take into account the impact of the presence of independent competing/interfering links (such as competing users in the unlicensed band) on the performance of the proposed method.

- In our model, although the cellular BS assists in CCD, each device is considered as an independent entity which acts to maximize its own payoff and can deviate from the proposed CCD method if a deviation increases its utility. We consider the impact of selfish strategies on the performance of the proposed method, i.e., the strategies in which a device deviates from the BS selection to maximize 
its payoff. One example of such strategies is the strategy where a selfish device is willing to receive contents from other devices via WiFi interface; however, to maximize individual payoff, it does not transmit content to other devices.

- We utilize the framework of repeated games with an infinite time horizon to model the interactions of users participating in CCD. We show that although the proposed SB method is efficient in terms of frequency carriers and energy savings performance, it is also vulnerable to selfish deviating users which poses a "tragedy of the commons" dilemma [14]. To address this challenge, we propose a carrier aggregation based incentive mechanism called the Follow-Reward and Disregard-Punish (FRDP) mechanism. The proposed mechanism rewards the devices that follow the BS selection by giving them higher cellular rates. Higher cellular rates are achieved using aggregation of those carriers that are saved due to CCD using the SB method. When a user is selected to broadcast the same received content and the user disregards the BS's selection, the user is punished in the next rounds by giving content to it only through a cellular interface with a single carrier.

- Finally, using the analytical and simulation results we show that the SB method with FRDP mechanism maximizes individual and network payoffs, and is also an equilibrium against unilateral selfish deviations.

The rest of the paper is structured as follows: In Section II, we review the related work. Section III presents the system setup and Section IV presents the proposed SB method. In Section V, a game theoretic formulation and stability analysis of the CCD using selected devices are presented. Section VI presents and evaluates a carrier aggregation based incentive mechanism for the SB method. The simulation results are then described in Section VII. Finally, Section VIII draws conclusions.

\section{RELATED WORK}

\section{A. Works based on theoretical and numerical analysis}

Broadly speaking, different methods of CCD using multiple interfaces can be divided into two categories: 1) Methods in which two or more devices that are in close proximity, simultaneously request the same content. Generally, in these methods the devices employ a primary interface (such as the cellular interface) for downloading the data content, and simultaneously use a secondary interface (such as WiFi or Bluetooth (BT)) for cooperatively sharing/distributing the content to other users near-by. 2) Methods in which two or more devices that are in close proximity, request the same content at different times. Generally, in such methods a device downloads the content using a primary interface and stores the content locally. A 
device then opportunistically exploits its secondary interface to either cooperatively deliver or download content to/from those devices that come in close contact and are interested in the same content. The implementation of opportunistic use of secondary interfaces is generally carried out taking into account factors, such as social ties among users, mobility, and infrastructure interactions.

In [5], [15], the authors consider CCD scenarios in which two or more users simultaneously request the same content at the same time. The works of [5], [15] propose a method called MicroDownload for CCD using multiple interfaces. In the proposed method, a device downloads part of video content from the server. The received content segment is then cooperatively broadcasted to other users in the group. The results in [5], [15] show improvement in performance of each device in terms of its video download rate (with no battery energy losses). In [16], the authors propose a CCD scheme using multiple interfaces, which is based on a network utility maximization (NUM) solution. The work in [16] considers those scenarios in which all smart devices in a group are used for CCD to maximize the video quality. A key feature of the CCD method in [16] is the use of network coding on downlink (from the source to the users), as well as on short range links between the users. The authors in [17] consider a scenario in which a group of users traveling in a train are interested in watching the same live video stream. The authors propose an approach in which every user using its cellular interface, downloads a subset of all video chunks, and simultaneously uses its short-range interface for coordination and group-internal redistribution of downloaded video chunks.

The performance of CCD methods using multiple interfaces can be affected by packet loss due to co-channel interference and channel errors. To preserve the content quality, the problem of lost packet recovery needs to be addressed. The works in [18], [19], [20] propose cooperative methods exploiting multiple interfaces for the recovery of lost content packets.

In most of the literature discussed above, the secondary links are considered to be similar in terms of their transmit/receive performances. In practical scenarios this is typically not true. Differences in link performances can have a strong impact on the overall performance of CCD method using multiple interfaces. To address this problem, our work in [21] proposes a device selection method for CCD that takes into account the link qualities of both the primary (cellular) and secondary link (WiFi/short-range) network interfaces. The work in [16] studies the device selection problem for non-real time applications, which are delay-tolerant in nature. It proposes a method in which the service provider delivers data content to only a small fraction of selected users through their cellular interfaces, and the selected users store the received data content in their own devices. The selected users are decided on the basis of their social 
ties. If social contacts of the selected users are within communication range of the selected users, and the social contacts request for the same stored content, the selected devices then use their secondary wireless interfaces to propagate the content further. The authors of [22] propose a framework called Push-and-Track which have designed for opportunistic vehicular networks. In this approach, a subset of users download data content using their cellular interface, and then starts propagating the same content epidemically, using their secondary interfaces. The mobile users which come in contact with any one of the subset of users can download the data content. In [23], the authors propose a community-based opportunistic dissemination method where the data content is distributed to only a subset of users addressed as initial sources, using cellular connections. This work focusses on how the initial sources are selected, on the basis of probability of encounters and their social relationship among one another, in the group. The initial sources are then allowed to propagate the content further through opportunistic communications. While the works in [16], [22], [23] present device selection methods for asynchronous content request scenarios, different from these works, our work presents a device selection method where multiple users simultaneously request the same content.

In this paper, we build on our previous research on CCD using multiple interfaces of selected devices [21]. Unlike the work in [21], we do not assume that each device always cooperatively follows the proposed method. This research takes into account the possibility of deviation by the selected users from participating in CCD. We formulate the problem of CCD using multiple interfaces of selected devices as a repeated game, and show that the method proposed in [21] is vulnerable to selfish deviations. To address this challenge, in our work we propose a carrier aggregation based incentive/punishment mechanism.

\section{B. Works based on measurement studies}

As discussed above, using theoretical and numerical analysis several different works have shown that CCD using multiple interfaces can improve the content delivery performance of cellular networks. However, to evaluate the content delivery performance using multiple interfaces, real-time measurements using different interfaces for content delivery are equally important. Several different works have performed real measurements to evaluate the energy and throughput performance of different wireless interfaces for content delivery. The work in [24] presents a Bit-per-Joule performance study of WiFi and BT interfaces on smart mobile devices. The results indicate that in the absence of co-channel interference, the performance of WiFi interface is better in terms of sending and receiving content, as compared to the performance of BT interface. When interference from WiFi or BT sources are taken into account, the performance of WiFi interface degrades, however, it still performs better in terms of sending and receiving content, as 
compared to the performance of BT interface. The authors of [25] present a measurement study of energy consumption characteristic of three widespread mobile networking technologies: 3G, GSM and WiFi. The results show that, on downloading a file of fixed size, the energy consumption of WiFi interface is less than the energy consumption of 3G and GSM interfaces. Another measurement study based on the energy consumption of three wireless communication technologies: BT, WiFi and 3G, is presented in [26]. The results show that for downloading a file of a fixed size, the energy consumption of WiFi interface is less than the energy consumption of BT and GSM interfaces. The work in [26] also shows that WiFi average data rate is higher than the $3 \mathrm{G}$ cellular average rate. The work in [27] makes a comparison of energy consumption of LTE, WiFi and interface aggregation of LTE and WiFi using Multipath TCP (MCTCP), on a mobile device. The results in [27] show that for downloading a file of a fixed size, energy consumed by a WiFi interface is less than the energy consumed by an LTE and aggregate (LTE and WiFi) interfaces. Finally the work of [28] conducts a measurement study regarding the energy consumed by a mobile device for downloading a YouTube content, using WDCDMA and WiFi interfaces. The results imply that energy consumption of WiFi interface is less than the energy consumption of cellular WCDMA interface.

In summary, the above discussed measurement based works point to the following conclusion: In terms of content delivery, WiFi performs better in terms of average energy efficiency and average content delivery rate as compared to different cellular and BT technologies.

\section{S YSTEM MODEL}

\section{A. Network model}

We consider a generic circular cellular cell of radius $\rho_{T}$ in which the BS is located at the centre of the circle. There are $N$ users in the cell who are interested in the same content. Let $\mathcal{N}=\{1,2,3, \cdots, N\}$ represent the set of $N$ users interested in the same content. The location of each user $i$, where $(i \in \mathcal{N})$, can be represented by the coordinates $\left(d_{i}, \theta_{i}\right)$, where $d_{i}$ is the distance between the BS and user $i$, and $0 \leq \theta_{i} \leq 2 \pi$. Two different distributions of $N$ users are considered within the cellular cell: 1) $N$ users are randomly distributed around the BS (see Fig. 1a); and 2) $N$ users are distributed in clusters around the BS. We consider that there are $n_{c}$ clusters in the cellular cell and each cluster has $n_{t}$ users within it such that $n_{c} * n_{t}=N$ (see Fig. 1b).

\section{B. Channel model}

Under the scenarios where the default mode is utilized for content delivery, a user independently downloads content using its own cellular connection. For instance, if all $N$ users utilize the default mode 
TABLE I: Notations

\begin{tabular}{|c|c|c|c|}
\hline Symbol & Meaning & Symbol & Meaning \\
\hline$\alpha$ & Path loss exponent & $n_{c}$ & Number of clusters in the cell \\
\hline$g_{f}$ & Fading gain & $n_{t}$ & Number of users in each cluster \\
\hline$\kappa$ & Path loss constant & $N_{S}$ & Average number of saved carriers \\
\hline$\theta_{i}$ & $0 \leq \theta_{i} \leq 2 \pi$ & $N_{D}$ & Number of users given content directly by BS \\
\hline$A_{c}$ & $\begin{array}{l}\text { Number of other competing WiFi users on same } \\
\text { channel as a user } i\end{array}$ & $N_{0}$ & Noise power density \\
\hline$B_{C}$ & Bandwidth of a cellular carrier & $P_{C, i}$ & Transmit power of cellular BS for user $i$ \\
\hline$B_{W}$ & Bandwidth of a WiFi channel & $P_{W_{t}, i}$ & Transmit power of device $i$ \\
\hline$d_{i}$ & Distance between BS and user $i$ & $P_{W_{r}, i}$ & Power utilized by secondary link for user $i$ \\
\hline$d_{i j}$ & Distance between user $i$ and user $j$ & $r_{i}^{C}, r_{i}^{N}, r_{i}^{R}$ & $\begin{array}{l}\text { Relative rate of user } i \text { when candidate, default and } \\
\text { recipient respectively }\end{array}$ \\
\hline$e_{i}^{C}, e_{i}^{N}, e_{i}^{R}$ & $\begin{array}{l}\text { Relative energy cost of user } i \text { when candidate, } \\
\text { default and recipient respectively }\end{array}$ & $\hat{r}_{i}^{C}, \hat{r}_{i}^{N}, \hat{r}_{i}^{R}$ & $\begin{array}{l}\text { Relative rate of user } i \text { when candidate, default and } \\
\text { recipient respectively with CA }\end{array}$ \\
\hline$\hat{e}_{i}^{C}, \hat{e}_{i}^{N}, \hat{e}_{i}^{R}$ & $\begin{array}{l}\text { Relative energy cost of user } i \text { when candidate, } \\
\text { default and recipient respectively with CA }\end{array}$ & $R_{C, i}$ & Average rate when user $i$ receive by cellular \\
\hline$E_{C, i}$ & $\begin{array}{l}\text { Average energy incurred by user } i \text { on receiving by } \\
\text { cellular }\end{array}$ & $\hat{R_{C, i}}$ & $\begin{array}{l}\text { Average rate when user } i \text { receive by cellular, with } \\
\text { CA }\end{array}$ \\
\hline$\hat{E_{C, i}}$ & $\begin{array}{l}\text { Average energy incurred by user } i \text { on receiving by } \\
\text { cellular, with CA }\end{array}$ & $R_{W_{r}, i}$ & $\begin{array}{l}\text { Average rate obtained by user } i \text { on receiving by } \\
\mathrm{WiFi}\end{array}$ \\
\hline$E_{N}, E_{S}$ & $\begin{array}{l}\text { Average energy cost per user in default cellular and } \\
\text { proposed method }\end{array}$ & $R_{W_{t}, i}$ & $\begin{array}{l}\text { Average rate obtained by user } i \text { on transmitting by } \\
\mathrm{WiFi}\end{array}$ \\
\hline$E_{W_{r}, i}$ & $\begin{array}{l}\text { Average energy incurred by user } i \text { on receiving by } \\
\mathrm{WiFi}\end{array}$ & $\rho_{C}$ & Radius of clusters in cell \\
\hline$E_{W_{t}, i}$ & $\begin{array}{l}\text { Average energy incurred by user } i \text { on transmitting } \\
\text { by WiFi }\end{array}$ & $\rho_{S}$ & Coverage radius of selected device \\
\hline$h_{i}$ & Channel gain between BS and user $i$ & $\rho_{T}$ & Radius of cellular cell \\
\hline$h_{i j}$ & Channel gain between user $i$ and user $j$ & $T_{C}$ & Average download time (default cellular) per user \\
\hline$I$ & Number of other competing interferers in a zone & $T_{S}$ & $\begin{array}{l}\text { Average download time (proposed method) per } \\
\text { user }\end{array}$ \\
\hline$J_{C}$ & $\begin{array}{l}\text { Average bit-per-Joule performance (default cellu- } \\
\text { lar) per user }\end{array}$ & $U$ & Average utility in a round \\
\hline$J_{S}$ & $\begin{array}{l}\text { Average bit-per-Joule performance (proposed } \\
\text { method) per user }\end{array}$ & $w_{1}, w_{2}$ & User's preference weights of metrics \\
\hline$N$ & Number of users in the cell & $X$ & $\begin{array}{l}\text { Number of circular zones of active independent } \\
\text { WiFi users }\end{array}$ \\
\hline
\end{tabular}

then the BS needs to have $N$ independent parallel sessions or carriers. Consider the case where the cellular BS sends a content segment to user $i$, we quantify the rate obtained as the Shannon capacity of the $i$ th link. The instantaneous cellular rate at time $t$ of the $i$ th link $R_{C, i}(t)$ can be expressed as

$$
R_{C, i}(t)=B_{C} \log _{2}\left(1+\frac{h_{i}(t) P_{C, i}}{B_{C} N_{0}}\right),
$$




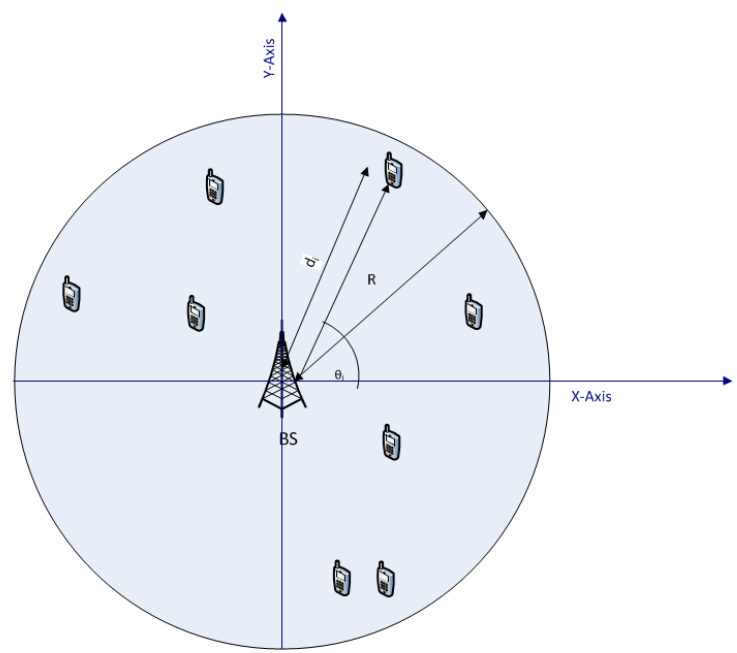

(a)

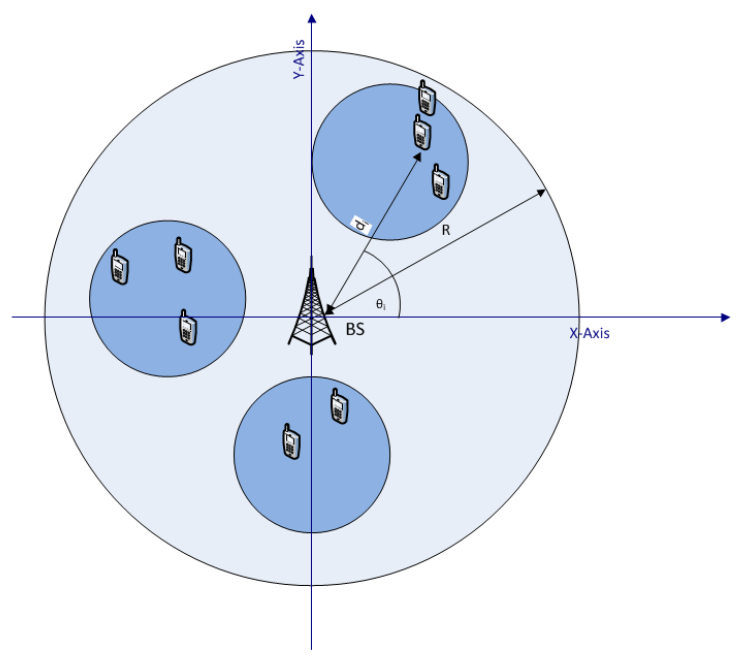

(b)

Fig. 1: a) A snapshot of random distribution of devices interested in the same same content; and b) A snapshot of clustered distribution of devices interested in the same same content.

where $h_{i}(t)$ is the channel gain at the time $t$ between the BS and user $i$, and is given as $h_{i}(t)=\frac{\kappa g_{f}(t)}{\left(d_{i}^{\alpha}\right)}$ with $\kappa$ being the path loss constant, $\alpha$ is the path loss exponent, $d_{i}$ is the distance between the BS and user $i$, $g_{f}(t)$ is the fading gain at the time instant $t . P_{C, i}$ is the transmit power of the cellular BS, $B_{C} N_{0}$ is the noise power and $B_{C}$ is the bandwidth of a carrier utilized by the BS to deliver the content to user $i$.

When multiple wireless interfaces of a selected device is utilized for CCD (based on device selection method as explained in the next section) by the BS, the selected device first downloads a part of the content using its cellular interface, and then using its WiFi interface it broadcasts the same received content part to the other users which are within its vicinity. The selected device receives the content using the cellular interface so its instantaneous rate at the time instant $t$ is also given by Eq. (1).

The success of receiving the content via WiFi link depends on the channel conditions between a selected broadcasting user and the receiving user. We consider that each selected device has a coverage radius of $\rho_{S}$. If user $j$ is within the coverage radius of any selected device, the data content segment can be successfully received. Moreover, the WiFi channel on which selected users broadcast can be shared by other independent active users or access points which in turn can lead to uncoordinated competition for channel access and also may lead to interference among users. To take this into account, we evaluate the performance of the considered CCD methods for two different scenarios: 1) When there is no other independent active user or access point on the WiFi channel which is used by the selected user; and 2) When other independent active users or access points are also using the same channel as a selected device. For simplicity, we consider that the other competing WiFi users are fully loaded. In such scenarios, a selected device $i$ can still expect to get its 'fair share' $\frac{1}{A_{c}+1}$ of the airtime when it is contending with the competing user, where $A_{c}$ is the number of other independent competing WiFi users on the same channel 


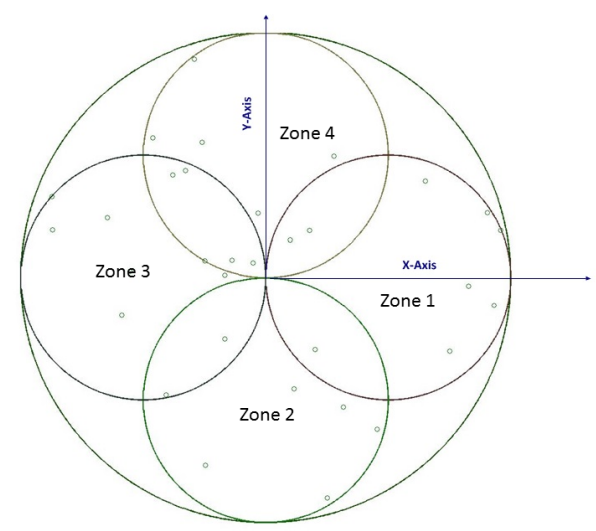

Fig. 2: A snapshot of distribution of other independent users that may compete with the considered $N$ users on a WiFi channel.

as user $i$ (see [29] for details where channel access share of WiFi users under fully loaded scenario is studied). In practice, WiFi users are in general clustered as the WiFi access points are mostly deployed in areas with higher user density such as residential areas, universities and hospitals. To take this into account, we consider that there are $X$ circular regions or zones of active independent WiFi users within the cellular cell, where $1 \leq X \leq 4$, and in each region there are $I$ competing WiFi users that are active (see Fig. 2). The instantaneous rate at time $t$, between a selected device $i$ and a device $j$ which receives the content from device $i$ via WiFi, can be expressed as

$$
R_{W_{r}, j}(t)=\left\{\begin{array}{l}
\left(\frac{1}{A_{c}+1}\right) B_{W} \log _{2}\left(1+\frac{h_{i j}(t) P_{W_{t}, i}}{B_{W} N_{0}}\right), \text { if } d_{i j} \leq \rho_{S} \text { for any } i \in \mathcal{N}_{c}, A_{c} \geq 0 \\
0, \text { otherwise, }
\end{array}\right.
$$

where $B_{W}$ is the bandwidth of the WiFi channel that is utilized by a selected device $i, P_{W_{t}, i}$ is the transmit power of selected device $i, h_{i j}(t)=\frac{\kappa g_{f}(t)}{\left(d_{i j}^{\alpha}\right)}$ is the channel gain with $\kappa$ being the path loss constant, $\alpha$ the path loss exponent, $d_{i j}$ the distance between the $i$ th selected device and the receiving user $j, g_{f}(t)$ is the instantaneous fading gain, $B_{W} N_{0}$ is noise power and $\mathcal{N}_{c}$ is the set of selected users.

\section{Performance Evaluation Metrics}

In this subsection, we present the considered metrics that are used for evaluating/comparing the performance of the proposed method.

1) Savings in terms of Cellular Carriers Utilized: Lets consider that $N$ users are interested in a (similar) content file. Denote $N_{D}$ as the number of carriers utilized when the cellular BS delivers the content to the $N_{D}$ users directly. Then average savings in the number of carriers per content file at the BS is given as

$$
N_{S}=N-\frac{\sum_{i=1}^{I_{N}} N_{D, i}}{I_{N}}
$$

Note that $I_{N}$ is number of times the experiment is conducted. 
2) Bits-per-Joule Performance: The average bit-per-Joule performance of a user when the cellular BS delivers a content file directly to the $N$ users is given as

$$
J_{C}=\frac{1}{N} \sum_{i=1}^{N} \frac{R_{C, i}}{P_{C, i}}
$$

where $R_{C, i}$ is the average rate of the $i$ th user and $P_{C, i}$ is the power utilized by the BS for that user. The average bit-per-Joule performance of a user when the cellular BS delivers the content to the $N_{D}$ users directly whereas $N-N_{D}$ users receive the content via WiFi interface is given as

$$
J_{S}=\frac{1}{N_{D}}\left(\sum_{i \in \mathcal{N}_{D}} \frac{R_{C, i}}{P_{C, i}}\right)+\frac{1}{N-N_{D}}\left(\sum_{i \in \mathcal{N}-\mathcal{N}_{D}} \frac{R_{W_{r}, i}}{P_{W_{r}, i}}\right),
$$

where where $\mathcal{N}_{D}$ is the set of users to whom the cellular BS delivers the content directly, $R_{C, i}$ is the average rate of the $i$ th user that is served by the BS directly, $P_{C, i}$ is the power utilized by the BS for that user, $R_{W_{r}, i}$ is the average rate of the $i$ th user that is served by the secondary link, $P_{W, i}$ is the power utilized by a secondary link for that user.

3) Average Time to Download a File: The third metric that we consider is average time to download a content file segment of size $S$ MBytes. Average time to download for a user when the cellular BS delivers content directly to the $N$ users is given as

$$
T_{C}=\frac{S}{N}\left(\sum_{i=1}^{N} \frac{1}{R_{C, i}}\right)
$$

Average time to download for a user when the cellular BS delivers the content to the $N_{D}$ users directly whereas $N-N_{D}$ users receive the content via WiFi interface is given as

$$
T_{S}=\frac{S}{N_{D}}\left(\sum_{i \in \mathfrak{N}_{D}} \frac{1}{R_{C, i}}\right)+\frac{S}{\left(N-N_{D}\right)}\left(\frac{S}{N_{D}} \sum_{i \in \mathfrak{N}_{D}} \frac{1}{R_{C, i}}+\sum_{i \in \mathcal{N}-\mathcal{N}_{D}} \frac{1}{R_{W_{r}, i}}\right) .
$$

4) Average Energy Cost: The average energy consumed to download a content segment of size $S$ when the cellular BS delivers the content file directly to the $N$ users is given as

$$
E_{N}=\frac{S}{N}\left(\sum_{i=1}^{N} \frac{P_{C, i}}{R_{C, i}}\right)
$$

The average energy consumed to download a content segment of size $S$ when the cellular BS delivers the content to the $N_{D}$ users directly, and the $N-N_{D}$ users receive the content via WiFi interface is given as

$$
E_{S}=\frac{S}{N_{D}}\left\{\sum_{i \in \mathfrak{N}_{D}}\left(\frac{P_{C, i}}{R_{C, i}}+\frac{P_{W_{t}, i}}{R_{W_{t}, i}}\right)\right\}+\frac{S}{\left(N-N_{D}\right)}\left(\sum_{i \in \mathcal{N}-\mathfrak{N}_{D}} \frac{P_{W_{r}, i}}{R_{W_{r}, i}}\right) .
$$

\section{Asymmetric Energy Consumption and Limited Battery of Devices}

In the scenarios where the best devices out of the total $N$ devices are selected by a BS for CCD, a user can be in one of the following operating modes. 

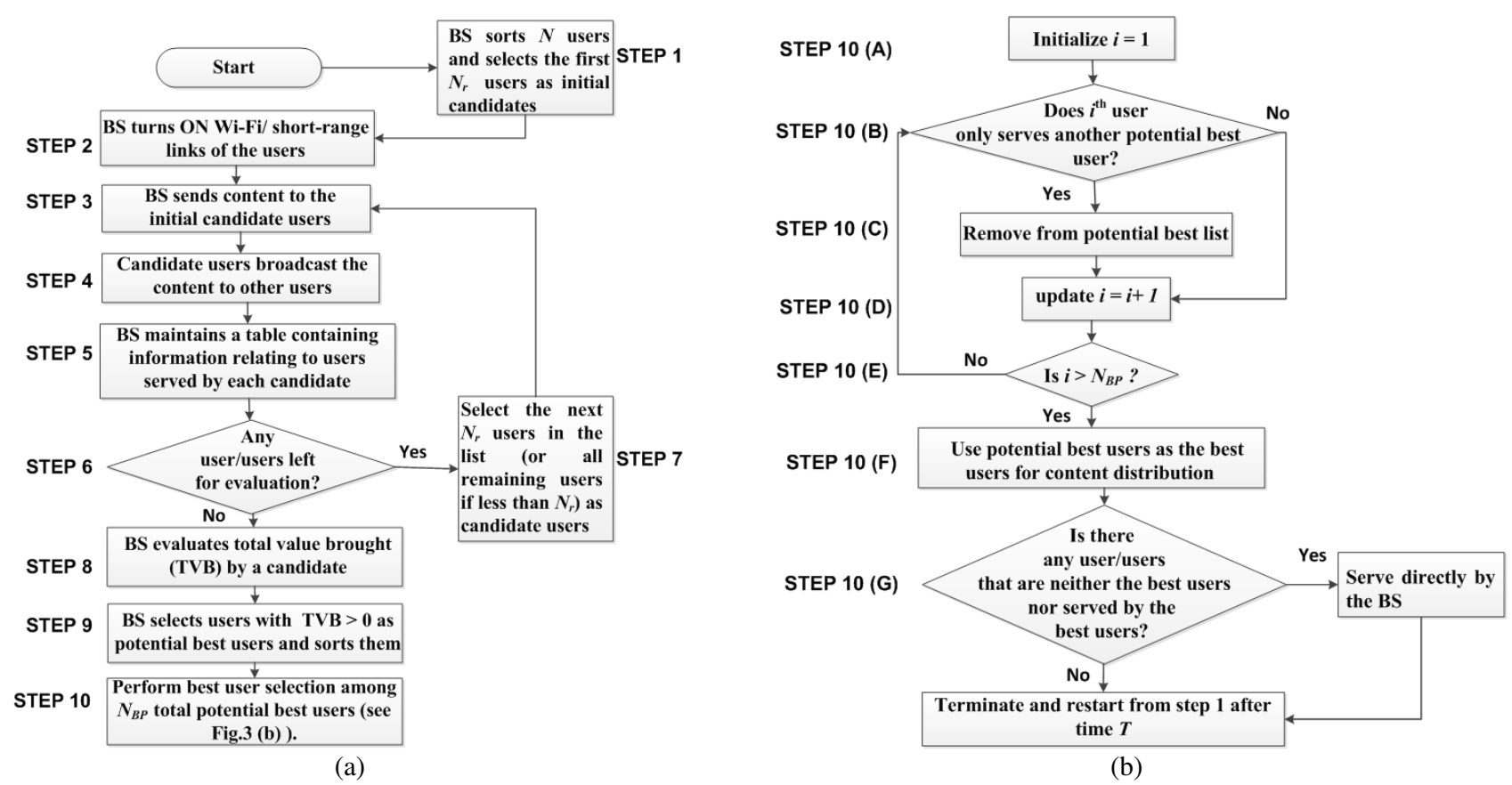

Fig. 3: The select best method for selection of best content delivery devices.

- Default mode: In CCD, there are users which are chosen neither as selected nodes nor as recipient nodes, probably due to their location and/or poor link conditions. Such nodes download the content directly from the BS.

- Selected mode: The BS selects a subset of users applying the proposed method for CCD (see the next section for details) that requires these users to contribute their battery resources in broadcasting the same content to other users. Selected users expend their battery energy on receiving the desired content through their cellular link with the BS. The users also expend their battery energy in broadcasting the same content using WiFi. Hence, the average energy consumption for a user when it is in selected mode is greater than when it is in default mode. However, the average rate obtained by a user when it is in the selected mode is equal to the average rate obtained when it is in the default mode.

- Recipient mode: In CCD there are users which receive broadcasted content from the selected nodes via their WiFi interface. The recipient users expend their battery energy in receiving the desired content via WiFi interface. As shown in many measurement papers (which were discussed in Section II) that WiFi outperforms cellular interface in terms of average energy and rate, hence, the average energy consumption for a user when it is in the recipient mode is less than when it is in the default mode. Moreover, the average rate obtained by a user when it is in the recipient mode is higher than the average rate obtained by the user when it is in the default mode. 
Given different energy costs incurred by a user when it is in the default, selected and recipient modes, it is useful to quantify the relative energy cost and relative rate of a user participating in CCD to the energy cost and rate, respectively, incurred by the user when it operates in the default cellular mode. We denote $e_{i}^{C}, e_{i}^{R}$, and $e_{i}^{N}$ to represent the relative energy costs incurred by a user $i$ being a selected, recipient, and default mode user, respectively. Similarly, $r_{i}^{C}, r_{i}^{R}$, and $r_{i}^{N}$ are used to represent the relative rates obtained by a user $i$ being a selected, recipient, and default mode user, respectively. When user $i$ is in the selected mode, the relative energy cost for user $i$ is given as

$$
e_{i}^{C}=-\frac{E_{C, i}}{E_{C, i}}-\frac{E_{W_{t}, i}}{E_{C, i}}=-1-\frac{E_{W_{t}, i}}{E_{C, i}}
$$

where $E_{C, i}$ is the average energy cost incurred by user $i$ when it is given content directly by the BS through the cellular interface and $E_{W_{t}, i}$ is the average energy cost incurred by user $i$ when it broadcasts content through the WiFi interface. The relative rate for user $i$ in the selected mode is expressed as

$$
r_{i}^{C}=\frac{R_{C, i}}{R_{C, i}}=1
$$

When user $i$ is in the recipient mode, the relative energy cost is given as

$$
e_{i}^{R}=-\frac{E_{W_{r}, i}}{E_{C, i}}
$$

where $E_{W_{r}, i}$ is the average energy cost when user $i$ receives the broadcasted content through the WiFi interface; and the relative rate for user $i$ in the recipient mode is expressed as

$$
r_{i}^{R}=\frac{R_{W_{r}, i}}{R_{C, i}}
$$

where $R_{W_{r}, i}$ is the average rate when user $i$ receives the broadcasted content through the WiFi interface. When a user $i$ is in the default mode (neither in the selected mode or in the recipient mode), its relative energy cost is given as

$$
e_{i}^{N}=-\frac{E_{C, i}}{E_{C, i}}=-1
$$

Its relative rate in the default mode is expressed as

$$
r_{i}^{N}=\frac{R_{C, i}}{R_{C, i}}=1
$$

\section{Proposed Select Best Method, Performance Analysis, and Selfish Deviations}

In this section, we present our SB method for the selection of best wireless content delivery devices, we also present some results relating to its performance analysis and discuss the impact of the presence of selfish users in the network. 


\section{A. Select Best Method}

The proposed method is described in Fig. 3 and the steps involved in the proposed method are further explained in detail as follows.

- Step 1: The BS sorts $N$ users in terms of decreasing cellular link quality and selects the first $N_{r}$ users to evaluate as possible candidates for content delivery. Note that the number $N_{r}$ of candidate users that are utilized for selection in each round depends on the user density. For a large number of $N$ users, $N_{r}$ can be taken as higher but for small to medium values it is kept small.

- Step 2: The short-range/WiFi link of the users are turned on by the BS.

- Step 3: The BS delivers a content segment (packets) to the selected candidate users.

- Step 4: The candidate users broadcast the received content through WiFi links while the other users listen.

- Step 5: The BS maintains a table containing served user and the number of total users served (TUS) by each candidate as follows: Each served user sends to the BS the number of packets received (successfully) from a candidate user. Along with this, it also sends the candidate users' DEV-ID such as MAC address to identify the candidate user and also to distinguish between the numbers of packets successfully sent by more than one candidate users, if any. As a user can receive packets from more than one candidate user, if it is in the vicinity of all of them. The BS maintains a table for candidates in the network in which when a user is successfully served by a candidate it is given a value of 1 ; otherwise 0 . Moreover, the BS also stores the total number of users (TUS) served by a candidate (by summing all the 1's).

- Steps 6 and 7: The process is repeated for the next round of candidate users, if any.

- Step 8: The BS evaluates the total value brought (TVB) by a candidate user as follows: Initially TVB $=$ TUS value, the BS sequentially evaluates each candidate to check if a candidate user is serving another user/users that is/are already served by another user. If a user is already served by another user the BS decreases the TVB value of this candidate user. In other words since the candidate user is serving an already served user it does not brings any additional benefit in terms of content distribution, and hence its value is decreased. Moreover, if a user receives the same packets from two different candidate users, for example, due to overlapping coverage, this can increase overhead in terms of energy and delay. To give further insights into the impact of overlapping user coverage scenarios, we provide some examples in the subsequent subsection.

- Step 9: The BS selects the candidate users with TVB $>0$ as the potentially best candidate $\left(N_{B P}\right)$ 


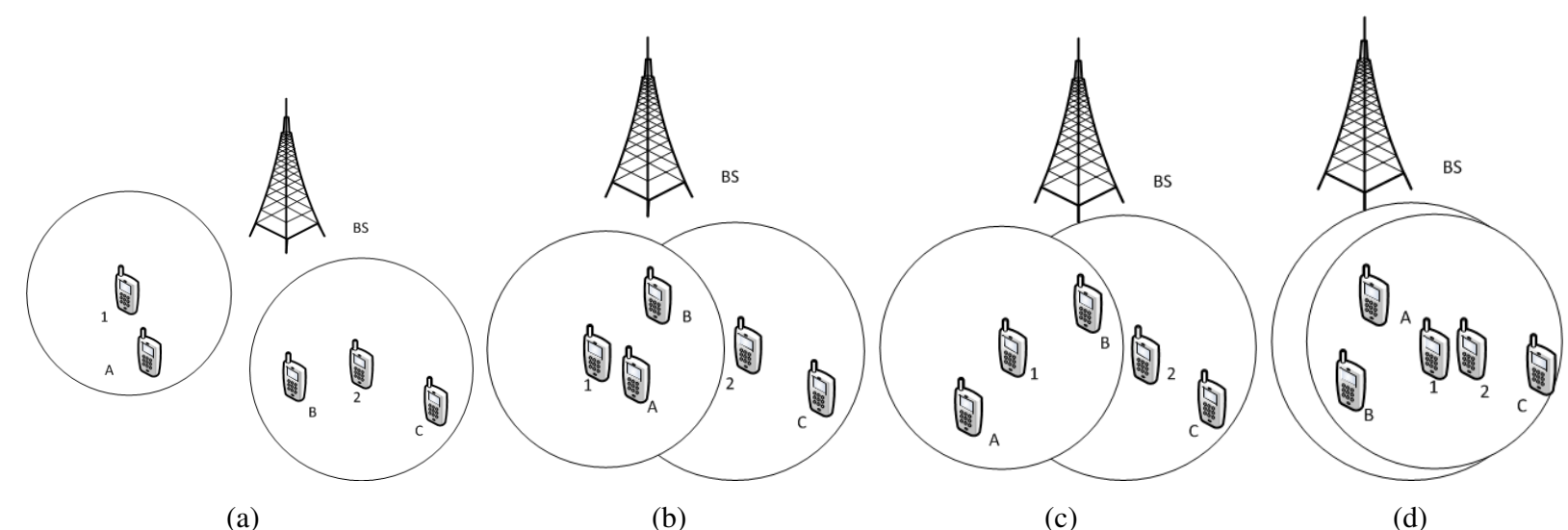

Fig. 4: Some example scenarios for coverage overlap between the selected users 1 and 2: a) Scenario where user 1 and 2 are chosen as selected users and their coverage is non-overlapping. b) Scenario where user 1 and 2 are chosen as selected users and their coverage is slightly overlapping. c) Scenario where user 1 and 2 are chosen as selected users and their coverage is partially overlapping. d) Scenario where user 1 and 2 are chosen as selected users and their coverage is almost completely overlapping.

users. It then sorts these users in terms of increasing cellular link quality.

- Step 10: The BS then checks that if any of the potentially best candidate users is only serving another potentially best candidate user, if yes, then it is removed from the list of selected users as this potentially best candidate user brings no additional value since it is serving another potentially best candidate user that is already served by the cellular link directly. Otherwise, the potentially best candidate user is chosen as the selected user. This process is repeated over all the potentially best candidate users. Hence, in this way, a group of selected users is decided to broadcast content. Finally, the BS checks if there is any user that is neither a selected user nor a recipient user. If there is any such user, the BS delivers the content directly to that user.

To incorporate changes in the interference environment and/or user distribution the process of evaluation of selected users is re-initiated after some time $T$. This time $T$ may be assigned according to variations in the interference environment or changes in the user distribution.

\section{B. Examples of coverage overlapping scenarios}

In this subsection, examples of coverage overlapping scenarios among the selected users are discussed and also illustrated in Fig. 4. In Fig. 4a, both the selected users, users 1 and 2 have non-overlapping WiFi coverage, due to which it is beneficial to select both the users to distribute content to different users, which in turn increases the coverage of CCD. In Fig. 4b, the WiFi coverage of the selected users 1 and 2 is slightly overlapping, where user 1 can serve A and B and user 2 can serve A, B and C. In such a case, it can be beneficial to select user 2 only for CCD, as user 1 brings no additional value in terms of user serving. In Fig. 4c, their WiFi coverage is partially overlapping, where user 1 is also in the coverage 


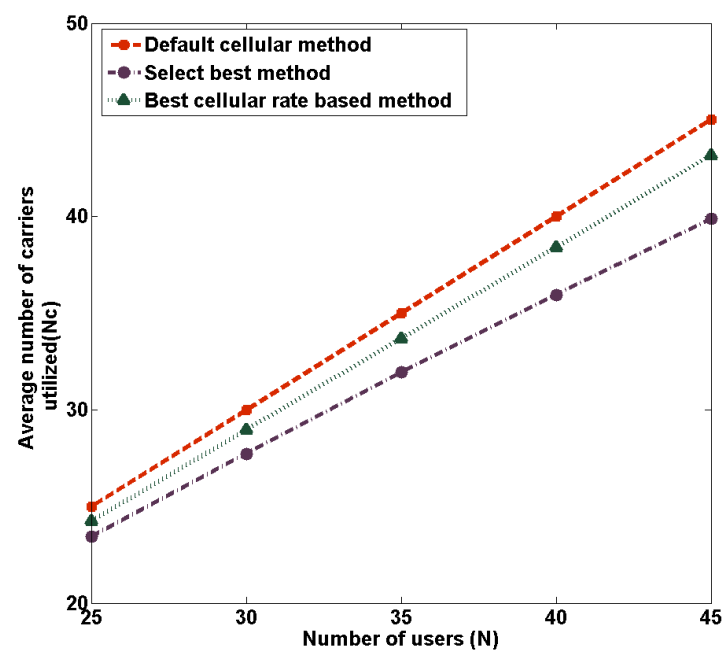

(a)

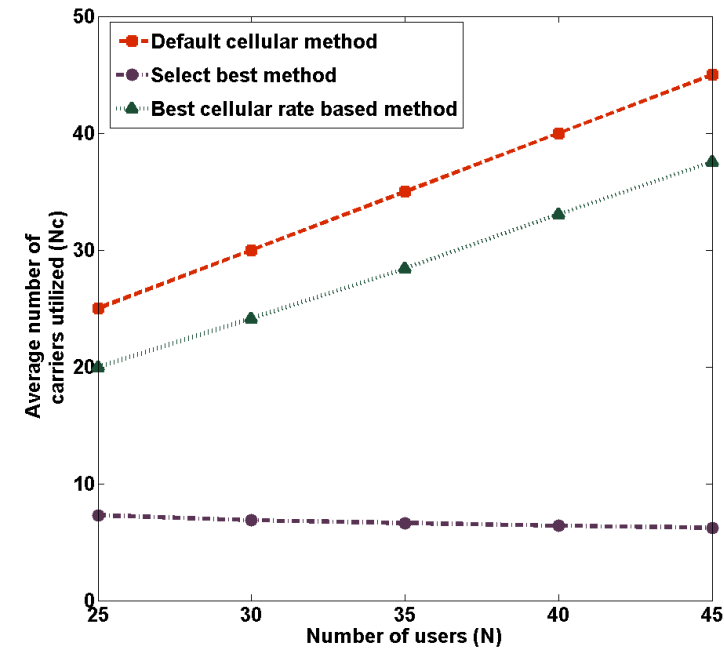

(b)

Fig. 5: Number of carriers vs number of users. (a) users are randomly distributed, (b) users are cluster-wise distributed

range of selected user 2 but not vice versa. However, both users 1 and 2 bring CCD benefit as user 2 can serve users $1, \mathrm{~B}$ and $\mathrm{C}$; user 1 can serve $\mathrm{A}$ and $\mathrm{B}$, where $\mathrm{A}$ is not served by user 2. In Fig. 4d, users 1 and 2 have WiFi coverage that is almost completely overlapping. In this case, the user that has a better cellular link quality should be chosen as the selected user.

\section{Performance Analysis}

We simulate a cellular cell of radius $\rho_{T}=1 \mathrm{~km}$ in which $N$ users that are interested in the same content are: 1) Randomly deployed; and 2) Deployed in clusters (see Section III for the details of user distribution). User clusters are generated by (randomly) dropping circles of radius $\rho_{C}$ in the cellular cell (see Fig. 1b). We assume that the transmitting power of the BS is $43 \mathrm{dBm}$ and the noise power is set to a value of $-100 \mathrm{dBm}$. Path loss values of $\kappa$ and $\alpha$ are set to 1 and 3 , respectively. The transmitting and receiving power of each user terminal is $20 \mathrm{dBm}$. The noise power is assumed to be $-40 \mathrm{dBm}$. The circular coverage region of each content delivery user (using WiFi link) is set to be 75 meters. The average cellular rates between the BS and a user is considered to be in the range of $600 \mathrm{Kbps}$ to $2 \mathrm{Mbps}$, and the average WiFi transmission rates among the users are considered to be in the range of 5 to $40 \mathrm{Mbps}$. We compare the performance of the proposed method for wireless content delivery against the following methods: 1) When only cellular link is used to deliver content to all the users; and 2) When the cellular BS selects a subset of users (based on best cellular link users) that cooperatively deliver content to the other users. Note that the process of content distribution has only one stage for method 1, i.e., where 


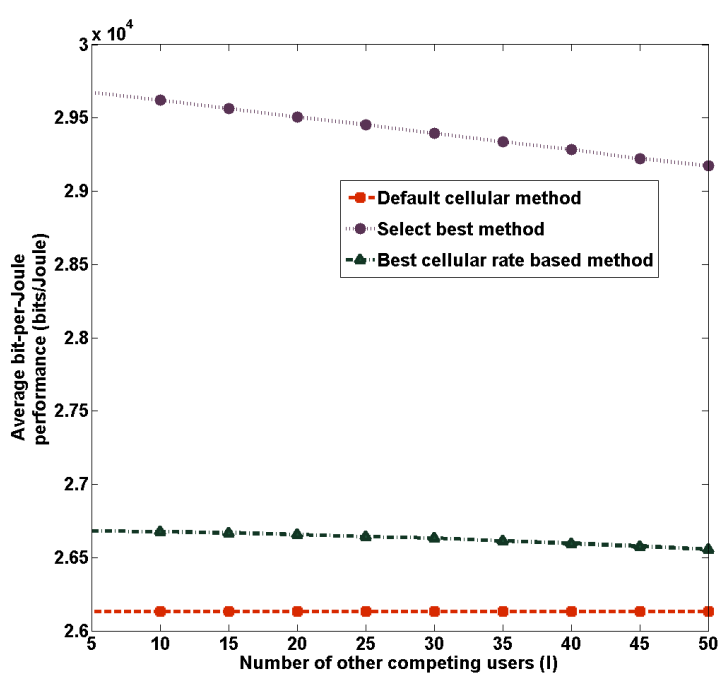

(a)

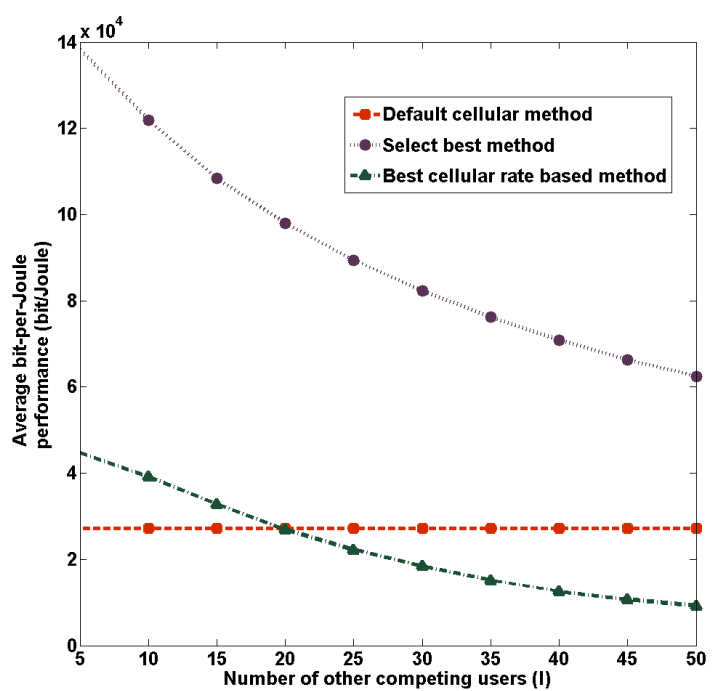

(b)

Fig. 6: Average bit-per-Joule performance vs number of other competing users. a) The users are randomly deployed; and b) the users are deployed in clusters.

the cellular BS delivers data to all users, whereas in method 2 and in the proposed method the content distribution process has two stages, i.e., where the cellular BS sends content to selected users (stage 1) via cellular link and then selected users broadcast data for other users via WiFi link (stage 2).

For analyzing the effect of other independent users operating in the same WiFi channel on the performance of the proposed method, we consider that there are $X$ circular regions in the cellular cell and in each these regions $I$ independent users operate. The values of $I$ are varied between 0 to 50 .

1) Number of frequency carriers employed: In Figs. 5a and 5b, we plot the average number of frequency carriers used by the BS (to deliver content to $N$ users) as a function of the number of $N$ users in the network under two different user distributions (for three different methods).

It can be seen that as expected the method in which only cellular link is utilized to deliver the content to $N$ users performs worst (in terms of number of carriers utilization). It can be also seen that when 50 active users are randomly distributed in the cell then the proposed method utilizes 7 carriers less than the default cellular method for content delivery. However, the real gains of the proposed method are evident in Fig. $5 \mathrm{~b}$ when the same 50 users have clustered distribution (10 clusters with 5 users in each cluster). The proposed method for clustered distribution requires significantly less carriers than the method that utilizes the default cellular method for content delivery.

2) Average bit-per-Joule performance: In Figs. 6a and 6b, for different scenarios, we present the average bit-per-Joule performance as a function of the number of other independent competing users 
in the cellular cell that utilize the same WiFi channel as the CCD devices. It can be seen that the proposed method achieves the highest bit-per-Joule performance as compared to the other two schemes when no other competing users are present on the WiFi channel. In the presence of other competing users, as expected, the conventional cellular method does not shows any change in performance and the cellular link-based selection method show little change in performance. On the other hand, although the performance of the proposed method degrades with increase in the number of other competing users, however, our proposed method still outperforms the other two methods.

\section{The SB method and selfish users}

CCD using the SB method requires users of selected devices to contribute their resources. A key challenge faced in the design of efficient CCD methods is that portable wireless devices have limited energy as they are battery powered. In our proposed SB method, although the cellular BS assists in CCD, each mobile user is an independent entity who acts to maximize its own utility and can deviate from the proposed CCD method if a deviation could increase its utility. In other words, the SB method can be vulnerable to free-riding users. One such example of free-riding in the SB method can be when some users when chosen as recipients, receive the broadcasted content from the other selected users but are not willing to contribute their own resources when they are selected to broadcast content to the other users. This motivates us to analyze the stability of the proposed SB method using the framework of repeated games. The framework of repeated games provides useful tools to study selfish behavior among CCD users that interact repeatedly over time to participate in content distribution [30], [31]. As the CCD users are unsure about when precisely their interactions with the other users will end, the model of repeated games with an infinite time horizon can be used to analyze such situations.

\section{Game Theoretic Formulation of CCD using Best Devices And its Analysis}

The CCD game using the best devices in a strategic form is specified by a 3-tuple $G=\left\langle\mathcal{N}, \mathcal{A}_{i}, U_{i}\right\rangle$, where

- $\mathcal{N}$ is a set of users that are interested in the same content within a macro-cell.

- A set of actions, $\mathcal{A}_{i}=\{F, D\}$, for each user $i \in \mathcal{N}$, that are taken when a user is either chosen as a selected mode or a recipient mode node. $F$ represents the action that a user cooperatively follow the BS selection, and $D$ represents the action that a user disregards the BS selection decision. The set of actions together define a set of possible action profiles $\mathbb{A}=\times_{i \in \mathcal{N}} \mathcal{A}_{i}$ and $\mathbf{a}=\left(a_{1}, a_{2}, \cdots, a_{N}\right)$ denotes a strategy profile of all users. 


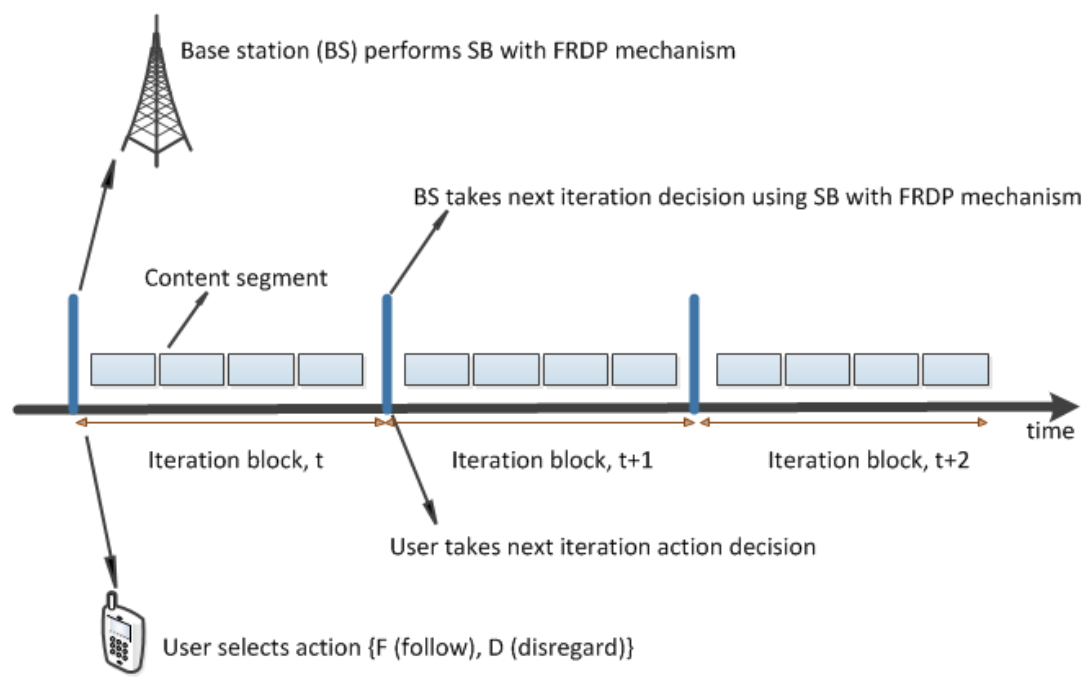

Fig. 7: CCD using best devices operation across multiple iterations (rounds).

- A utility function for each user $i$

$$
U_{i}=p_{c, i}\left(w_{1} e_{i}^{C}+w_{2} r_{i}^{C}\right)+p_{r, i}\left(w_{1} e_{i}^{R}+w_{2} r_{i}^{R}\right)+p_{n, i}\left(w_{1} e_{i}^{N}+w_{2} r_{i}^{N}\right)
$$

where

- $p_{c, i}$ and $p_{r, i}$ represent the probabilities of a user being in the selected mode and in the recipient mode, respectively. $p_{n, i}$ represents the probability that user $i$ is in the default cellular mode as explained earlier, where $p_{n, i}=\left(1-p_{c, i}-p_{r, i}\right)$.

- $e_{i}^{C}, e_{i}^{R}$, and $e_{i}^{N}$, represent the relative energy costs incurred by user $i$ (see Eqs. (10-15));

- $r_{i}^{C}, r_{i}^{R}$, and $r_{i}^{N}$ represent the relative rates obtained by a user; and

$-w_{1}$ and $w_{2}$ are user $i$ 's preference weights of metrics, where $w_{1}+w_{2}=1$. The choice of weights $w_{1}$ and $w_{2}$ may depend on the particular device's battery condition. For instance, for a user with a low battery level, the energy cost may be more important than the obtained rate. As a result, the higher weight may be assigned to the energy part of the utility as compared to the rate part. In the infinitely repeated game model of CCD using best devices, the stage game $G$ is played at each round $k$, where in each round content segments of the requested content are delivered. We consider that the users make decisions rationally to maximize their long-term expected utilities. The average utility per round of user $i$ is given by

$$
V_{i}=\lim _{\bar{T} \rightarrow \infty} \frac{1}{\bar{T}} \sum_{k=1}^{\bar{T}} U_{i}^{k}
$$

Next we establish the condition under which following the base station's selection, i.e., playing the action $F$, yields a higher average utility as compared to when each user downloads the content directly through 
an individual BS carrier.

Under the default method, where every user downloads content directly through a BS carrier, then in Eq. (16), $p_{c, i}=0, p_{r, i}=0$, and $p_{n, i}=1$. The average utility of user $i$ is independent of the actions of other users and in this case the average utility is given as

$$
U_{i}=w_{1} e_{i}^{N}+w_{2} r_{i}^{N}
$$

Note that for this case when $w_{1}=w_{2}$ then $U_{i}=-1 / 2+1 / 2=0$, as $e_{i}^{N}=-1$ and $r_{i}^{N}=1$. Under the proposed SB method when all users play $F$, i.e., $U_{i}\left(a_{i}, a_{-i}\right)=U_{i}(F,(F, F, \cdots, F))$, in each round then using Eq. (16), the average utility for each user $i$ in each round is

$$
\begin{aligned}
U_{i}\left(a_{i}, a_{-i}\right) & =U_{i}(F,(F, F, \cdots, F)) \\
& =p_{c, i}\left\{w_{1}\left(-1-\frac{E_{W_{t}, i}}{E_{C, i}}\right)+w_{2}\right\}+p_{r, i}\left\{w_{1}\left(-\frac{E_{W_{r}, i}}{E_{C, i}}\right)+w_{2}\left(\frac{R_{W_{r}, i}}{R_{C, i}}\right)\right\}+p_{n, i}\left(-w_{1}+w_{2}\right) .
\end{aligned}
$$

To ensure that playing the action $F$, i.e., cooperating and following the BS selection in each round, yields a higher average utility as compared to when the users download the content directly through a BS carrier, the average utility of each user when they play $F$ should be greater than the utility that users can obtain when they download content directly through the BS carrier, i.e.,

$$
U_{i}(F,(F, F, \cdots, F))>-w_{1}+w_{2}
$$

For $w_{1}=w_{2}=\frac{1}{2}$, by solving inequality (20), we obtain

$$
\frac{p_{c, i}}{p_{r, i}}<\left(\frac{R_{W_{r}, i} E_{C, i}-E_{W_{r}, i} R_{C, i}}{R_{C, i} E_{W_{t}, i}}\right) .
$$

In other words, to ensure a higher utility due to cooperation, for each user $i$ the ratio of probability of being served in the selected mode to the probability of being served in the recipient mode, i.e., $\frac{p_{c, i}}{p_{r, i}}$, must satisfy Eq. (21). Next we provide intuitive explanation of Eq. (21) by setting different values for relative rates and energy. We consider the following examples: 1) When average WiFi rate is twice as the average cellular rate, average energy consumed in transmitting content for a user in the selected mode using WiFi interface is twice as much as average energy consumed in receiving the same content by cellular interface, and the average energy consumed in receiving content for a user in the recipient mode using WiFi interface is half as much as energy consumed in receiving the same content by cellular interface. In this case, using Eq. (21), $\frac{p_{c, i}}{p_{r, i}}<0.75$. This means that for a user using the SB method, higher utility as compared to the utility obtained using the default cellular mode for a user can only be maintained when a user is more often in the recipient mode as compared to the selected mode. 2) When the energy values are the same 
as in the previous example but the $\mathrm{WiFi}$ rate is five times as cellular rate then $\frac{p_{c, i}}{p_{r, i}}<2.25$. This means that using the SB method, a higher utility can now even be maintained when a user more often serves as selected user as compared to a recipient. It is important to note that due to the broadcast nature of WiFi links a single user in the selected mode may be able to serve many recipients. The proposed SB method only selects a user to be in the selected mode when there is at least one recipient in its vicinity that can receive the content. Since in our model all users are treated equally, hence under the proposed method $\frac{p_{c, i}}{p_{r, i}} \geq 1$. Before presenting further results we next make the following observation.

Remark A deviation where user $i$ plays action $D$ when it is selected by the BS as a recipient is inefficient for that user. This follows from the fact that the average energy cost is less and the average data rate is higher when user $i$ receives content on its WiFi interface as compared to when it receives content directly from the cellular interface (as discussed earlier in Section II). Moreover, using our proposed SB method, a user is selected as a recipient only when a user with good WiFi link to the recipient is present in its vicinity. This ensures that only those WiFi links are utilized for cooperation that have good link quality.

The deviation from the proposed method that we need to consider is that deviation where a user $i$ is to serve as a selected mode user and it plays $D$. In this case by disregarding the BS selection, the user $i$ can save its energy costs that are incurred in broadcasting the content via its WiFi interface.

Next we show that in the proposed game the strategy profile where all users follow the SB selection i.e., play $F$ in each round, $\mathbf{a}=(F, F, F, \cdots, F)$, is not a Nash equilibrium (NE).

Proposition 5.1: In the proposed game, the strategy profile where all users follow the SB selection i.e., play $F$ in each round, $\mathbf{a}=(F, F, F, \cdots, F)$, is not a Nash equilibrium (NE).

Proof: When all users follow SB method selection, the outcome will be cooperation in each round, whose average utility per round for a user $i$ is $U_{i}(F,(F, F, \cdots, F)$ ) (given in Eq. (19)).

Consider the unilateral deviation where user $i$ plays $D$ in each round when it is chosen to be in the selected mode, otherwise it plays $F$, whereas all the other users play always $F$. Since all other users follow the SB method, user $i$ will obtain the same average utility, as before, in the rounds where it is in the recipient mode and also where it is neither in the recipient nor in the selected mode. However, in the rounds where user $i$ is in the selected mode, user $i$ can now save its energy costs that are incurred in broadcasting the content via its WiFi interface. The average utility per round for user $i$ now is

$$
\begin{aligned}
U_{i}(D,(F, F, \cdots, F)) & =p_{c, i}\left(-w_{1}+w_{2}\right)+p_{r, i}\left(w_{1} e_{i}^{R}+w_{2} r_{i}^{R}\right)+p_{n, i}\left(-w_{1}+w_{2}\right), \\
& =p_{c, i}\left(-w_{1}+w_{2}\right)+p_{r, i}\left\{w_{1}\left(-\frac{E_{W_{r}, i}}{E_{C, i}}\right)+w_{2}\left(\frac{R_{W_{r}, i}}{R_{C, i}}\right)\right\}+p_{n, i}\left(-w_{1}+w_{2}\right) .
\end{aligned}
$$

It is easy to see that $U_{i}(D,(F, F, \cdots, F))>U_{i}(F,(F, F, \cdots, F))$, which proves our claim. 
Proposition 5.1 shows that the proposed method is vulnerable to free-riding users and poses a "tragedy of the commons" dilemma [14]. This implies that if too many users exploit others' WiFi interfaces for content delivery, the excess of free-riders drives away the cooperating users that make the cooperation viable. Next, we present a carrier aggregation based incentivizing mechanism for the SB method that allows the users to cooperate and obtain a higher utility. We also show that under the proposed incentive mechanism following the SB method is a Nash Equilibrium.

\section{The Proposed CCD Game With CARrier Aggregation Based InCEnTives}

\section{A. Cooperation Stimulation Mechanisms}

The design of mechanisms aimed at incentivizing/punishing users in wireless networks to achieve cooperation is an extensively researched topic. The works in [32]-[35] have shown that the use of direct and indirect reciprocity can help mitigate the free-rider problem in wireless networks. We next define direct and indirect reciprocity as follows:

Definition 1: Direct reciprocity means there are repeated interactions between the same two users, and user $i$ 's behavior towards user $j$ depends on what user $j$ has done to user $i$. Indirect reciprocity means there are repeated interactions among a group of individuals, and user $i$ 's behavior towards user $j$ also depends on what user $j$ has done to others.

Although, the free-riding phenomenon is by no means unique to the proposed CCD method. However, the characteristics of the proposed CCD method present unique challenges and opportunities for the design of novel incentive and punishment mechanisms to ensure CCD. This is due to the reason that straight forward applications of well-known direct and indirect reciprocity mechanisms for incentivizing cooperation and punishing defections among users may not be effective in the context of the proposed method. For instance, due to changes in cellular network topology, users involved in CCD changes over a period of time. A user that is currently involved in CCD with a user/group of users may have to later involve in CCD with some other user/group of users. Hence, very often a user may have no opportunities for direct/indirect incentivization, or retaliation in response to free riding, as user interactions change over a period of time. Moreover, in practice it is memory and computation intensive to follow a reciprocity based protocol that requires each user to observe and keep track of identities, strategies and reputation of different users with which they interact over a period of time. To avoid such memory and computation intensive operations on a user's device, we present a method in which the BS implements the proposed incentive and punishment mechanisms to discourage the users from the free-riding behavior. 


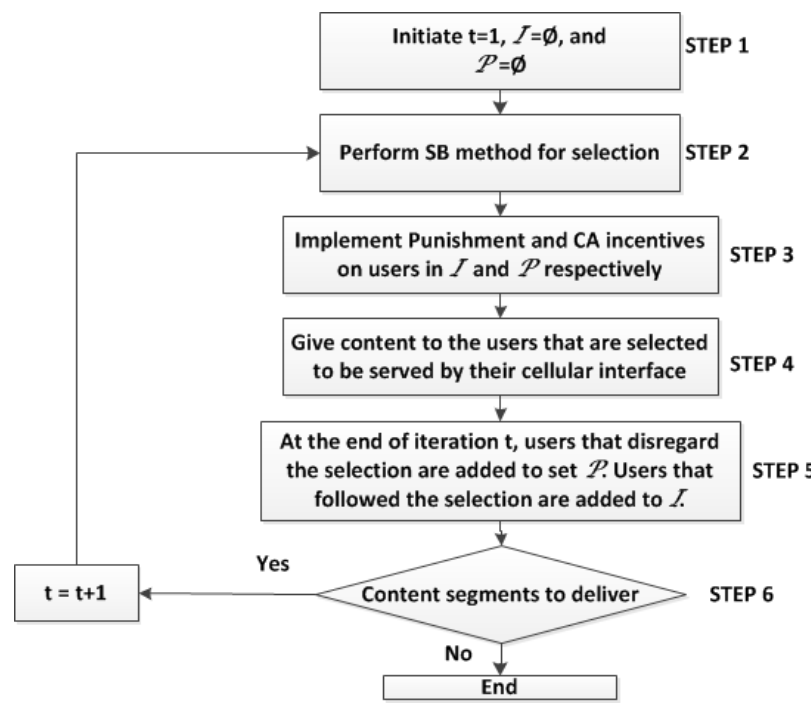

Fig. 8: Flow diagram of the FRDP incentive strategy

\section{B. The SB Method with FRDP Incentive Mechanism}

In this subsection, we propose the Follow-Reward and Disregard-Punish (FRDP) incentive mechanism for selfish users in CCD with the SB method. The flow diagram of the steps involved in the FRDP mechanism is given in Fig. 8. The core idea behind the proposed mechanism is as follows:

- When a user is chosen as a selected mode user and the user follows the BS selection, then the user is rewarded with higher cellular rate for content delivery. When it is selected to be served via cellular interface, the content is delivered at a higher rate to the user by aggregating the user's default cellular carrier with additional carriers that are saved due to the recipient users receiving content via WiFi link. The aggregation of carriers in cellular networks is commonly known as carrier aggregation (CA).

- When a user is chosen as a selected user and the user disregards the BS selection, then the user is punished. The BS punishes the user by delivering content to it through a cellular interface with a single carrier when it is selected to be served via WiFi interface.

The flow diagram of the incentive mechanism is illustrated in Fig. 8, and the steps involved in it are explained as follows.

- Step 1: Initialization of the iteration $(t=1)$, initialize $I=\emptyset$, as the set of users in CA incentive group, and initialize $\mathcal{P}=\emptyset$, as the set of users in punishment group.

- Step 2: The BS performs user selection applying the SB method in the beginning of every iteration $t$. We define an iteration $t$ as a block of content segment delivery periods of fixed duration during which the BS does not change the selection of users, and the users do not change their actions. FRDP 
operation across multiple iterations is illustrated in Fig. 7

- Step 3: After performing selection, the BS checks whether a user selected to be in the recipient mode is in the set $\mathcal{P}$. If yes, the BS punishes the user for the entire iteration block in the following ways. In the given iteration, if the user is selected to be served via WiFi interface, the BS punishes the user by delivering content to it through its cellular interface with a single carrier. In the given iteration, if the user is selected to be served via cellular interface then no CA is employed for the user. After the punishment, in which the user is served with a single cellular carrier when it was supposed to be served via the user's WiFi interface, the user is removed from the punishment group $\mathcal{P}$. However, if the user is selected to be delivered content via cellular link and it is in the set $I$, the BS then incentivizes the user (as explained above in the core principle) for the entire block, and then removes it from the CA incentive group $I$.

- Step 4 The BS delivers the content to the users that are to be served by their cellular interface.

- Step 5: The BS checks if the selected users followed the selection in the current iteration.

- Step 5(a): If a user follows it is added to the set of users $I$ in the CA incentive group.

- Step 5(b): If the selected user disregards the selection by the BS, then it is removed from the CA group and added to the set of users $\mathcal{P}$ in the punishment group.

- Step 6: The steps 2 till 5 of the FRDP strategy are repeated till the requested content is delivered to the users.

Under the proposed FRDP mechanism when all users always follow the SB method selection, the average utility of user $i$ is given as:

$$
\begin{aligned}
U_{i}^{F R D P}(F,(F, F, \cdots, F))= & p_{c, i}\left(w_{1} \hat{e_{i}^{C}}+w_{2} \hat{r_{i}^{C}}\right)+p_{r, i}\left(w_{1} \hat{e_{i}^{R}}+w_{2} \hat{r_{i}^{R}}\right)+p_{n, i}\left(w_{1} \hat{e_{i}^{N}}+w_{2} \hat{r_{i}^{N}}\right), \\
= & p_{c, i}\left\{w_{1}\left(-\frac{\hat{E_{C, i}}}{E_{C, i}}-\frac{E_{W_{t}, i}}{E_{C, i}}\right)+w_{2}\left(\frac{\hat{R_{C, i}}}{R_{C, i}}\right)\right\}+p_{r, i}\left\{w_{1}\left(-\frac{E_{W_{r}, i}}{E_{C, i}}\right)+w_{2}\left(\frac{R_{W_{r}, i}}{R_{C, i}}\right)\right\} \\
& +p_{n, i}\left\{w_{1}\left(-\frac{\hat{E_{C, i}}}{E_{C, i}}\right)+w_{2}\left(\frac{\hat{R_{C, i}}}{R_{C, i}}\right)\right\},
\end{aligned}
$$

where

- $\hat{e}_{i}^{C}, \hat{e}_{i}^{R}$, and $\hat{e}_{i}^{N}$ represent the relative energy costs incurred by user $i$ being chosen as a selected mode, a recipient mode and a default user, respectively, when there is CA incentive. All energy costs are defined relative to the energy costs when the user is given content only using the default cellular mode;

- $r_{i}^{C}, \hat{r_{i}^{R}}$, and $\hat{r}_{i}^{N}$ represent the relative rates obtained by a user being a selected, recipient and default user, respectively, when there is CA incentive. All rates are defined relative to the obtained rates 
when the user is given content only using the default cellular mode; and

- $\hat{E}_{C, i}$ represents the average energy cost and $\hat{R_{C, i}}$ represents the average rate of user $i$ when the user is given content directly by the BS through the cellular interface using CA.

To ensure that playing the action $F$ yields a higher average utility as compared to when the users download the content directly through a BS carrier, it is required that $U_{i}^{F R D P}(F,(F, F, \cdots, F))>-w_{1}+w_{2}$. Earlier in Section V we showed that for WiFi rate twice the cellular rate, using the SB method (with no FRDP incentive mechanism) ensures a higher utility only when, i.e., $\frac{p_{c, i}}{p_{r, i}}<0.75$, a user more often serves as a recipient user as compared to it serves as a selected user. By setting the same relative values in the above inequality, as the values used in Section $\mathrm{V}$ for inequality (21), it can be seen that $p_{c, i}<0.55 p_{r, i}+0.20$. In other words, for the same considered scenario using the SB method with FRDP incentive mechanism, for $p_{c, i}<0.20$ the user $i$ can obtain higher utility as compared to the default cellular mode even when $p_{r, i}=0$.

Proposition 6.1: In the proposed CCD game, when the SB method with the FRDP incentive mechanism is used for CCD then the strategy profile where all users value $w_{1} \leq w_{2}$ and play $F$ in each round, $\mathbf{a}=(F, F, F, \cdots, F)$, is a Nash equilibrium (NE).

Proof: When all users follow the SB method with FRDP incentive mechanism, the outcome will be cooperation in each round, whose average utility per round for user $i$ is given in Eq. (23). To show that the strategy profile $(F, F, F, \cdots, F)$ is a Nash equilibrium, we need to consider a deviating user $i$, whereas all other users play $F$. In the proposed SB method with FRDP incentive mechanism, when the user $i$ deviates in any given round then in the next few rounds (until the user is removed from the punishment group) the user can get punished in one of the following ways: a) In a round, if the user is selected to be served via WiFi interface, the BS punishes the user by delivering content to it through its cellular interface with a single carrier. b) In a round, if the user is selected to be served via cellular interface then no $\mathrm{CA}$ is employed for the user. After the punishment round in which the user is served with a single cellular carrier when it was supposed to be served via the user's WiFi interface, the user is then removed from the punishment group. To show that the SB method with FRDP mechanism is a Nash Equilibrium, we need to show that the sum of the obtained utilities in a deviating round and the subsequent rounds in which the user gets punished is less than the sum of the obtained utilities if the user has followed the strategy in the same rounds. Now, consider the deviation where the deviating user $i$ plays $F$ in the $\tau-1$ rounds then plays $D$ in the $\tau$ th round when the user is selected as a recipient user, and then again keep playing $F$ for the remaining rounds, whereas all the other users always play $F$. The long time expected utility of user $i$ is given as

$$
\sum_{k=1}^{k=\tau-1} U_{i}^{F R D P}(F,(F, F, \cdots, F))+U_{i}^{F R D P}(D,(F, F, \cdots, F))+\sum_{k=\tau+1}^{\infty} U_{i}^{F R D P}(F,(F, F, \cdots, F)) .
$$




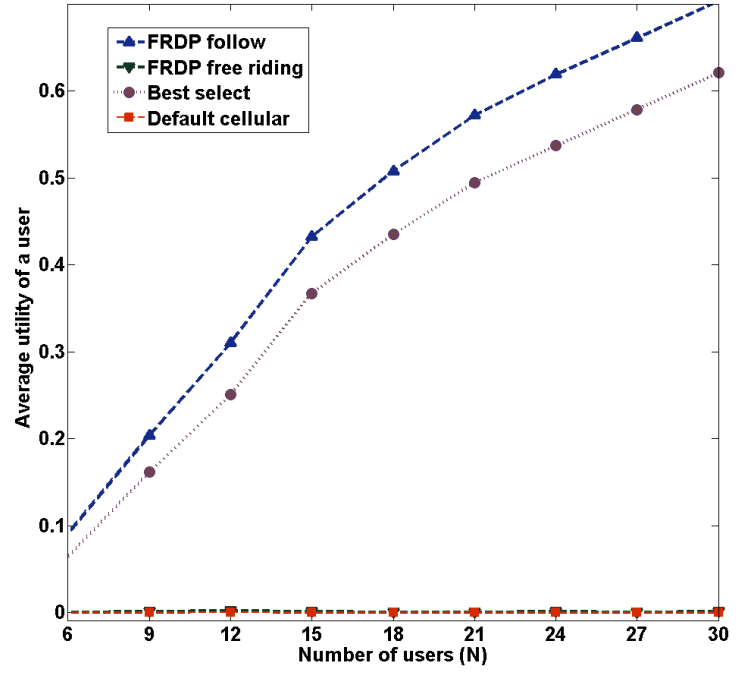

(a)

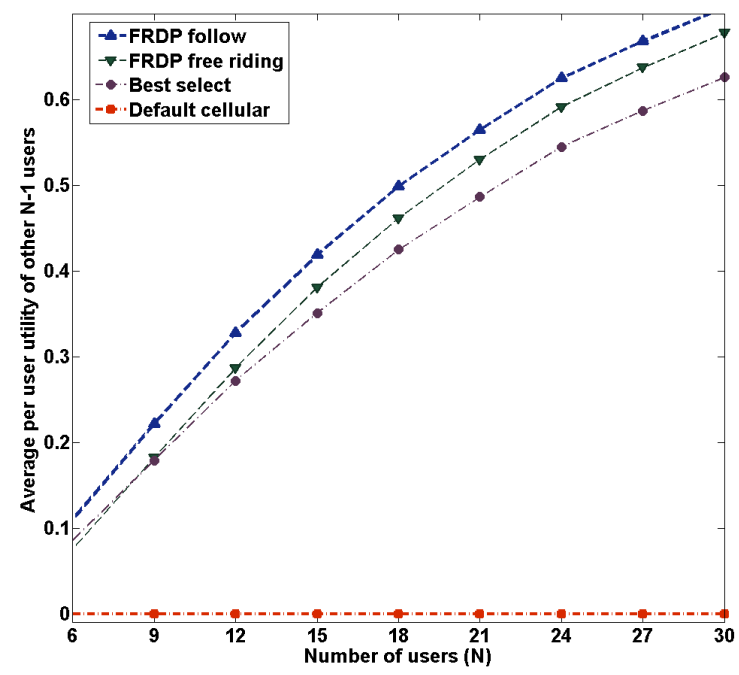

(b)

Fig. 9: Average utility of a) user $i$; and b) average per user utility of other $N-1$ users that always follow the CCD methods, as a function of increasing number of $N$ users. $w_{1}=w_{2}$ are set to be equal to 0.5 .

The average per round utility of each of other users that play $F$ in every round is given in Eq. (23). The average per round utility of the deviating user that plays $F$ in the first $\tau-1$ rounds is the same as of other users (given by Eq.(23)). In the $\tau$ th round the deviating user gains in utility by not delivering the content via WiFi interface, the average payoff for the deviating user $i$ in the $\tau$ th round is

$$
U_{i}^{F R D P}(D,(F, F, \cdots, F))=\left\{w_{1}\left(-\frac{\hat{E_{C, i}}}{E_{C, i}}\right)+w_{2}\left(\frac{\hat{R_{C, i}}}{R_{C, i}}\right)\right\} .
$$

In the next round, i.e., $(\tau+1)$ th round, the BS punishes the user and the user's utility in each of the next few rounds (until the user is removed from the punishment group) is $-w_{1}+w_{2}$. Let us consider the best possible case for the deviating user $i$ in which the user $i$ after deviating is punished only for one round. This best case scenario happens, when after deviating, in the next round the user is selected as a recipient mode user and is served via cellular interface instead of WiFi interface, the user is then removed from the punishment group. For the two rounds, in which the user deviates in the first and gets punished in the second round, the average sum utility of the user is $\left[w_{1}\left(-\frac{E_{C, i}}{E_{C, i}}\right)+w_{2}\left(\frac{R_{C, i}}{R_{C, i}}\right)+\left(w_{2}-w_{1}\right)\right]$. Instead of deviating, if the user had followed the strategy then the user's average sum utility would have been $\left[w_{1}\left(-\frac{E_{C, i}}{E_{C, i}}-\frac{E_{W_{t}, i}}{E_{C, i}}\right)+w_{2}\left(\frac{R_{C, i}}{R_{C, i}}\right)+w_{2}\left(\frac{R_{W_{r}, i}}{R_{C, i}}\right)-w_{1}\left(\frac{E_{W_{r}, i}}{E_{C, i}}\right)\right]$. It can be seen that the user saves energy in the first round when it deviates, however, due to punishment it loses in terms of both energy and rate in the second round, as it is not given content via WiFi interface. On average WiFi rates are higher than the cellular rates and also on average WiFi has less energy costs. Moreover, unlike the best case scenario 
discussed above, the deviating user can also get punished by not getting higher cellular rates via CA incentive. Hence, the average sum utility due to deviation is less than the sum utility obtained when the user follows the strategy. In other words, there is no incentive in unilateral deviation, which proves our claim.

It is important to note that for the scenarios where $w_{1}>w_{2}$, i.e., where users value energy more than their obtained rates, the proposed strategy is still Nash equilibrium when the difference between WiFi energy/rates and cellular energy/rates are much higher as compared to the difference between $w_{1}$ and $w_{2}$, and/or when the users are given a higher number of carriers as CA incentives for CCD.

\section{Simulation Results}

Using the same simulation parameters as used in Section IV-C, in this section, we evaluate the performance of the proposed FRDP mechanism for the SB method under different scenarios. In our simulations, we consider two different behaviors of selected users for CCD: a) a behavior in which a user always follow with the BS selection; and b) a behavior in which a user always free rides, i.e., it disregards the BS selection when it is selected to broadcast the content to other users. We compare the performance of the proposed mechanism with the following other methods: 1) The method in which only cellular link is used to deliver content to all users in each iteration; and 2) The method in which the cellular BS utilizes the proposed SB method but the FRDP mechanism is not employed by the BS.

\section{A. Performance in terms of Average utility as a function of $N$ users}

In Figs. 9a and 9b, we compare the average utility obtained by a user $i$ with average per-user utility of other $N-1$ users that always follow the CCD methods for four different methods. It can be seen that in terms of average utility, the performance of the user $i$ when it free rides is significantly less as compared to when it always follow the SB method with FRDP mechanism. On the other hand, for the scenario where the user $i$ free-rides, the average per-user utility performance of the other $N-1$ users who always follow the SB method with FRDP mechanism is better than the free riding user. Moreover, it can be also seen in the two figures that the scenario where all $N$ users always follow the proposed SB method with FRDP mechanism performs better as compared to other scenarios.

\section{B. Performance in terms of Average utility as a function of $w_{1}$}

In Fig. 10a, for different scenarios we present the average utility obtained by a user $i$ as a function of the user's preference weight $w_{1}$, i.e., the user's preference weight for energy costs. The preference weight 


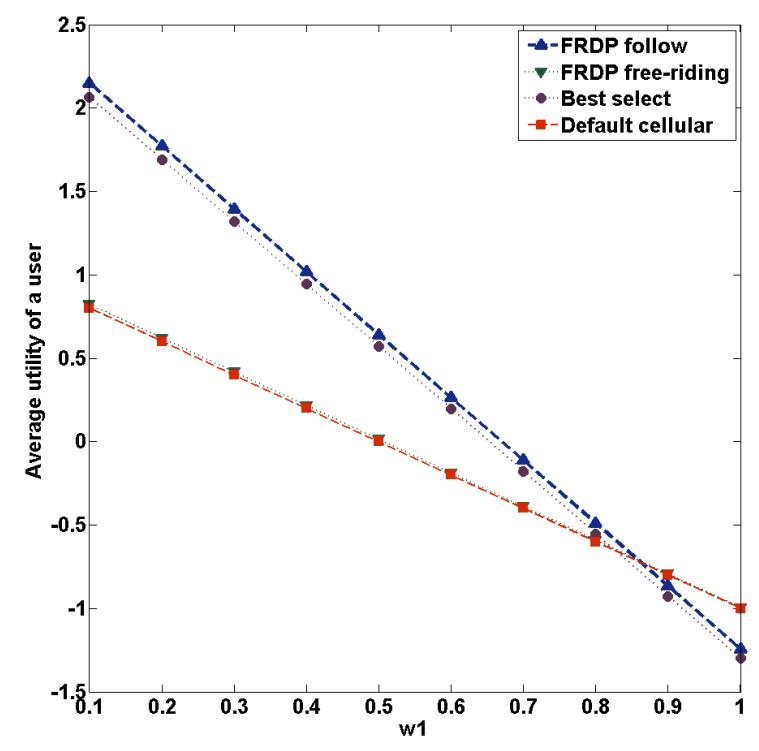

(a)

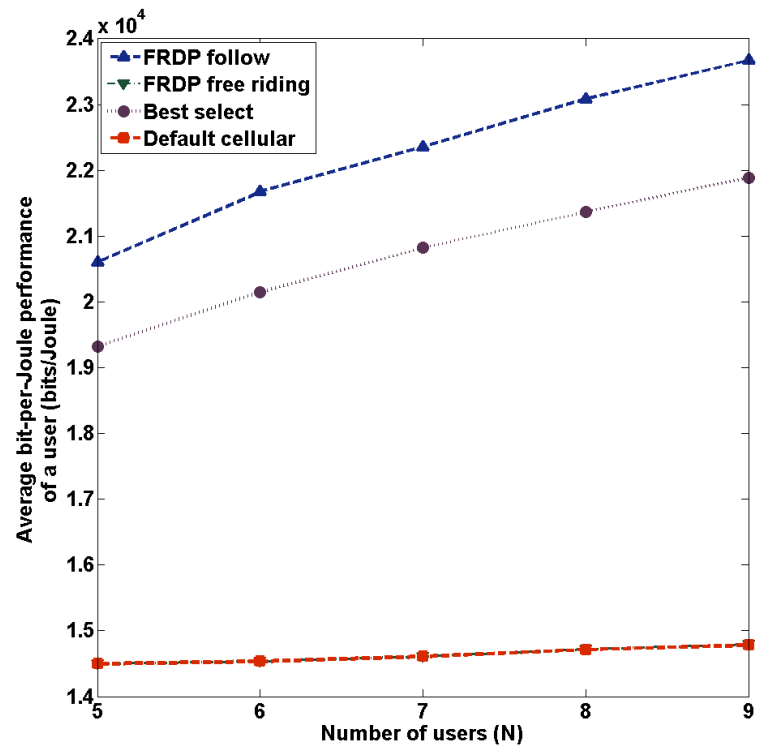

(b)

Fig. 10: a) Average utility of a user as a function of $w_{1}$; and b) average bit-per-Joule performance of a user vs number of users.

$w_{1}$ may depend on a particular device's battery condition. In Fig. 10a, it can be seen that when the user's preference weight $w_{1}$ for energy cost is small, then as compared to the other methods the average utility obtained by the user $i$ is significantly higher for the scenario in which all the users always follow the SB method with FRDP incentive mechanism. It can be also seen that for high preference weights such as $w_{1}=0.85$, and $w_{2}=0.15$, the proposed SB method with FRDP mechanism performs the same as the other methods. We note that in practice, a user may prefer to have very high preference weight for energy costs when its battery energy is very low. In such scenarios it may not be efficient to select users with low batteries for CCD. In practice, there are softwares available that can report the battery condition of a device to the BS, and the BS using the proposed SB method with FRDP mechanism can take this into account by simply not selecting those users that have low battery energy.

\section{Performance in terms of Average bit-per-Joule performance as a function of $N$ users}

In Fig. 10b, we plot the average bit-per-Joule performance of user $i$ as a function of number of users $N$, for different methods. It can be seen that in terms of the average bit-per-Joule performance the user performs best for the method in which all users follow the SB method with FRDP mechanism. Moreover, it can be also seen that the user's performance is significantly less for the method that utilizes only cellular link to deliver content to all users. 


\section{CONCLUSION}

To address the problem of cellular network congestion in the context of content delivery to multiple users simultaneously, in this paper, we studied the use of multiple wireless interfaces for CCD. Efficient CCD methods that utilize multiple interfaces need to intelligently use these interfaces to ensure that interfaces with good link conditions are used so as to avoid the usage of interfaces during their potential periods of bad link conditions. We showed that our proposed SB method which selects devices with high link quality for content distribution, leads to energy savings for devices and frequency carrier savings for a cellular base station.

Mobile-to-mobile CCD requires users to contribute their resources, such as battery energy and device computation resources. Although a cellular BS assists in CCD, however, each mobile user is an independent entity who acts to maximize its own utility, and can deviate from the CCD method if a deviation could increase its utility. This motivated us to analyze the stability of the proposed SB method against selfish deviations using the framework of repeated games with an infinite time horizon. We showed that although the proposed SB method is efficient in terms of frequency carrier and energy savings performance, however, it is not an equilibrium against a selfish deviating user. To address this problem, we proposed a carrier aggregation based incentive mechanism called Follow-Reward and Disregard-Punish (FRDP) mechanism for the SB method. The proposed mechanism rewards the users that follow the BS selection by giving them higher cellular rates, using aggregation of those carriers that are saved due to $\mathrm{CCD}$ in a given round of CCD. When a user is selected to broadcast the same received content and the user disregards the BS selection, then the BS punishes it in the next rounds by giving content to it through a cellular interface with a single carrier. Our analytical and simulation results have shown that the SB method with FRDP mechanism maximizes individual and network payoffs, and is stable against unilateral selfish deviations.

\section{REFERENCES}

[1] HUAWEI, "Future Smartphone Solution White Paper," 2012.

[2] Ericsson, "5G Radio Access," 2013.

[3] W. paper Qualcomm, "Rising to Meet the 1000x Mobile Data Challenge," 2012.

[4] J. Andrews, S. Buzzi, W. Choi, S. Hanly, A. Lozano, A. Soong, and J. Zhang, "What Will 5G Be?, IEEE Journal on Selected Areas in Communications, vol. 32, pp. 1065-1082, June 2014.

[5] A. Le, L. Keller, H. Seferoglu, B. Cici, C. Fragouli, and A. Markopoulou, "MicroCast: Cooperative Video Streaming using Cellular and D2D Connections," CoRR, vol. abs/1405.3622, 2014.

[6] K. K. Yap, T. Y. Huang, Y. Yiakoumis, S. Chinchali, N. McKeown, and S. Katti, "Scheduling Packets over Multiple Interfaces While Respecting User Preferences," in Proceedings of the Ninth ACM Conference on Emerging Networking Experiments and Technologies, Santa Barbara, California, USA, CoNEXT '13, pp. 109-120, December 2013. 
[7] S. Sharafeddine, K. Jahed, N. Abbas, E. Yaacoub, and Z. Dawy, "Exploiting Multiple Wireless Interfaces in Smartphones for Traffic Offloading," in Communications and Networking (BlackSeaCom), 2013, (Georgia), pp. 142-146, July 2013.

[8] D. M. West, "Mobile learning: Transforming education, engaging students, and improving outcomes,"

[9] H. Seferoglu, L. Keller, B. Cici, A. Le, and A. Markopoulou, "Cooperative Video Streaming on Smartphones," in Communication, Control, and Computing (Allerton), 2011 49th Annual Allerton Conference on, (Monticello, IL), pp. 220-227, September 2011.

[10] H. Bo, H. Pan, V. S. A. Kumar, M. V. Marathe, S. J. A., and Srinivasan, "Mobile Data Offloading Through Opportunistic Communications and Social Participation,” IEEE Transactions on Mobile Computing, vol. 11, pp. 821-834, May 2012.

[11] S. Ioannidis, A. Chaintreau, and L. Massoulie, "Optimal and Scalable Distribution of Content Updates Over a Mobile Social Network," in INFOCOM 2009, IEEE, Rio de Janeiro, Brazil, pp. 1422-1430, April 2009.

[12] J. Whitbeck, M. Amorim, Y. Lopez, J. Leguay, and V. Conan, "Relieving the Wireless Infrastructure: When Opportunistic Networks Meet Guaranteed Delays," in World of Wireless, Mobile and Multimedia Networks (WoWMoM), 2011 IEEE International Symposium in Lucca, Italy, June 2011.

[13] T. Chen and M. D. Katz, "Cooperative Architechture for Cellular-short-range Combined Mesh Networks," in Proceedings of the 5th International ICST Mobile Multimedia Communications Conference, London, United Kingdom, Mobimedia '09, September, 2009.

[14] G. Hardin, “The Tragedy of the Commons,” Science, vol. 162, pp. 1243-1248, December 1968.

[15] K. Lorenzo, L. Anh, and C. Blerim, "MicroCast: Cooperative Video Streaming on Smartphones," Cisco Systems. Cisco visual networking index: Global mobile data traffic forecast update, 2011-2016.

[16] B. Han, P. Hui, V. Kumar, M. Marathe, J. Shao, and A. Srinivasan, "Mobile Data Offloading through Opportunistic Communications and Social Participation," Mobile Computing, IEEE Transactions on, vol. 11, pp. 821-834, May 2012.

[17] M. Stiemerling and S. Kiesel, “A System for Peer-to-peer Video Streaming in Resource Constrained Mobile Environments," in Proceedings of the 1st ACM Workshop on User-provided Networking: Challenges and Opportunities, U-NET '09, pp. 25-30, December 2009.

[18] L. Shenjie and S. H. G. Chan, "BOPPER: Wireless Video Broadcasting with Peer-to-Peer Error Recovery," in IEEE International Conference on Multimedia and Expo, Beijing, July 2007.

[19] L. Xin, G. Cheung, and C. Chuah, "Rate-distortion Optimized Network Coding for Cooperative Video Stream Repair in Wireless Peerto-Peer Networks," in Proceedings of International Symposium on a World of Wireless, Mobile and Multimedia Networks (WoWMoM), Newport Beach, CA, June 2008.

[20] P. Vingelmann, M. Pedersen, F. Fitzek, and J. Heide, "On-the-Fly Packet Error Recovery in a Cooperative Cluster of Mobile Devices," in Proceedings of Global Telecommunications Conference (GLOBECOM), Houston, TX, December 2011.

[21] Barua, Bidushi and Khan, Zaheer and Han, Zhu and Latva-aho, Matti and Katz, Marcos, "On the Selection of Best Devices for Cooperative Wireless Content Delivery," in Global Communications Conference (GLOBECOM), 2014 IEEE, Austin, TX, December 2014.

[22] "Push-and-Track: Saving Infrastructure Bandwidth Through Opportunistic Forwarding," Pervasive and Mobile Computing, vol. 8, no. 5, pp. $682-697$, October, 2012.

[23] Y.-J. Chuang and K.-J. Lin, “Cellular Traffic Offloading Through Community-based Opportunistic Dissemination,” in Wireless Communications and Networking Conference (WCNC), Shanghai, April 2012.

[24] R. Friedman, A. Kogan, and Y. Krivolapov, “On Power and Throughput Tradeoffs of WiFi and Bluetooth in Smartphones," Mobile Computing, IEEE Transactions on, vol. 12, pp. 1363-1376, July 2013.

[25] N. Balasubramanian, A. Balasubramanian, and A. Venkataramani, "Energy Consumption in Mobile Phones: A Measurement Study and Implications for Network Applications," in Proceedings of the 9th ACM SIGCOMM Conference on Internet Measurement Conference, (Chicago, Illinois, USA), pp. 280-293, November 2009. 
[26] G. Kalic, I. Bojic, and M. Kusek, "Energy Consumption in Android Phones when using Wireless Communication Technologies," in MIPRO, 2012 Proceedings of the 35th International Convention, (Opatija), pp. 754-759, May 2012.

[27] Lim, Yeon-sup and Chen, Yung-Chih and Nahum, Erich M. and Towsley, Don and Gibbens, Richard J., "How Green is Multipath TCP for Mobile Devices?," in Proceedings of the 4th Workshop on All Things Cellular: Operations, Applications, \& Challenges, AllThingsCellular '14, Chicago, Illinois, USA, pp. 3-8, August 2014.

[28] Y. Xiao, R. Kalyanaraman, and A. Yla-Jaaski, "Energy Consumption of Mobile YouTube: Quantitative Measurement and Analysis," in Next Generation Mobile Applications, Services and Technologies, 2008. NGMAST '08, (Cardiff), September 2008.

[29] P. Bahl, R. Chandra, T. Moscibroda, R. Murty, and M. Welsh, "White Space Networking with Wi-Fi like Connectivity,” Proceedings of the ACM SIGCOMM 2009 conference on Data communication in Barcelona, Spain, vol. 39, no. 4, pp. 27-38, August 2009.

[30] A. Rubinstein, Modeling Bounded Rationality. The MIT Press, Cambridge MA, 1997.

[31] J. Romero, "Finite Automata in Undiscounted Repeated Games with Private Monitoring,” Purdue University Economics Working Papers, Purdue University, Department of Economics, 2011.

[32] Yan Chen and Liu, K.J.R., "Indirect Reciprocity Game Modelling for Cooperation Stimulation in Cognitive Networks," IEEE Transactions on Communications, vol. 59, pp. 159-168, January 2011.

[33] Yan Chen and Liu, K.J.R., "Cooperation Stimulation in Cognitive Networks Using Indirect Reciprocity Game Modelling," in Global Telecommunications Conference (GLOBECOM 2010), Miami, FL, December 2010.

[34] M. Piatek, T. Isdal, T. Anderson, A. Krishnamurthy, and A. Venkataramani, "Do Incentives Build Robustness in Bit Torrent?," in Proceedings of the 4th USENIX Conference on Networked Systems Design \& Implementation in Cambridge, MA, NSDI'07, 2007.

[35] B. Niu, H. Zhao, and H. Jiang, "A Cooperation Stimulation Strategy in Wireless Multicast Networks," IEEE Transactions on Signal Processing, vol. 59, pp. 2355-2369, May 2011. 